\title{
A BRIEF INTERNET-BASED TREATMENT FOR PANIC DISORDER
}

\author{
Britt Klein and Jeffrey C. Richards \\ University of Ballarat, Australia
}

\begin{abstract}
This study investigated the effectiveness of an Internet-based intervention for people with panic disorder. Twenty-two participants met criteria for panic disorder and were randomly assigned to either the treatment or a self-monitoring control condition. The study took place over 3 weeks and consisted of one week of self-monitoring prior to the intervention group accessing the program for one week, followed by one week of post-intervention assessment. Participants were assessed on measures pertaining to panic, negative affect, body vigilance, anxiety sensitivity and self-efficacy in managing panic. The treatment condition was associated with significant reductions in all variables except anxiety sensitivity and depressive affect. This type of intervention for people with fearful panic attacks holds promise as an efficacious and economical method for treating spontaneous panic.
\end{abstract}

Keywords: Panic disorder, Internet-based treatment, cognitive behaviour therapy.

\section{Introduction}

Various studies from around the world have confirmed cognitive behaviour therapy as the treatment of choice for panic disorder. Self-help methods appear to offer promise for increasing the accessibility and affordability of this treatment. For example, Gould and Clum (1995) found bibliotherapy and individual therapy to be equally effective for panic disordered patients over 4 weeks and at 2-month follow-up. Bibliotherapy has also been shown to be as effective as group cognitive behaviour therapy at 6-month follow-up. Hecker, Losee, Fritzler and Fink (1996) also found self-directed cognitive behavioural intervention to be as effective as therapist-directed treatment at 6-month follow-up. Nevertheless, although there appears little difference in outcome between interventions utilizing therapist-directed CBT and self-help methods, the latter routinely include some therapist-assistance and involvement.

Recently, computer technology has been developed as an adjunct to treatment for anxiety disorders (Newman, Consoli, \& Taylor, 1997). Newman, Kennardy, Herman and Taylor (1997) found a therapist-assisted 4-session palmtop computer program was as effective as 12 sessions of individual treatment for panic disorder at 6-month follow-up. The cost of their palmtop computers at $\$ 500$ may, however, be prohibitive for many people. Although

Reprint requests and requests for extended report to Jeffrey C. Richards, School of Behavioural \& Social Science and Humanities, University of Ballarat, PO Box 663, Ballarat, Vic 3353, Australia.

E-mail: j.richards@ballarat.edu.au 
computer technology shows promise in assisting therapist-directed treatment, it is unclear how useful this technology may be in self-help interventions. Important considerations are cost of the equipment and its potential to engage panickers to become involved in the program with minimal therapist involvement.

The aim of the present study was to develop and evaluate an Internet-based self-help treatment program incorporating the principles of cognitive therapy for people with panic disorder. We reasoned that the use of this medium would involve minimal cost for the participant (many people in Australia have access to the Internet and we were also able to provide such access for people at the University); and that such a program would enable us to design an engaging, flexible program that would facilitate completion with little therapist assistance.

\section{Method}

\section{Participants}

Twenty-three people with a diagnosis of panic disorder were drawn from either regional Victoria (52\%) or from Melbourne (48\%). One participant failed to complete the study and subsequently her data were excluded. Of the remaining 22 participants, 19 were female and 3 were male. Average age for women was 40 years $(S D=13.29)$, and 46 years for men $(S D=20.29)$; average education level (years of schooling) was 11.7 years $(S D=2.02)$. Nine participants $(41 \%)$ reported the use of anxiety medication but subsequently all reported no alteration in their dosage levels throughout the 3 weeks. All participants reported no contact with any mental health professional for the duration of the study.

Criteria for inclusion were a primary diagnosis of panic disorder and absence of any significant physical health problems. All participants had experienced spontaneous panic attacks in the past 12 months and indicated at least one of the following: fear of future panics, worry about the consequences of panic attacks, or a significant change in behaviour as a consequence of the attacks. These variables were assessed by the Prime-MD as well as 8-point rating scales, based upon those in the Anxiety Disorders Interview Schedule for DSM-IV where a rating greater than 4 is clinically significant. Although the mean frequency of panic attacks experienced at the pre-intervention assessment was relatively low, at least one of the above three criteria for the diagnosis of panic disorder reached clinical significance (i.e., had a rating of 4 or more) for all participants. Therefore, although most participants were not frequent, severe panickers, all had a diagnosable panic disorder by DMS-IV criteria.

\section{Measures}

Prime MD. This is a brief, structured interview for the diagnosis of anxiety and mood disorders, somatoform and eating disorders, and alcohol abuse/dependence.

Panic Attack Record From. Participants rated strength of each panic attack experienced over the 3 weeks of this study on a scale from 0 (none) to 8 (extreme) and recorded each symptom experienced during the panic attack. Additional information included duration of panic, where it occurred, how stressful the panic was and whether it was expected. 
Daily Record Form. Participants recorded their daily average fear of panic, average depression and anxiety levels using scales from 0 (none) to 8 (extreme).

Self Efficacy Questionnaire. This questionnaire consists of six pairs of statements, each pair referring to a different source of efficacy in being able to manage panic attacks. They included perceived difficulty in managing panic, amount of effort to interrupt panic attacks, and perceived competence in interrupting panic once it has started.

Body Vigilance Scale. This is a 4-item self-report measure of attentional focus on internal bodily sensations. Items measure the degree of attentional focus on, and perceived sensitivity towards changes in bodily sensations, and the amount of time spent scanning for bodily sensations.

Anxiety Sensitivity Index. This is a 16-item self-report measure of fear of autonomic arousal symptoms.

\section{Design and procedures}

The first component of the Internet-based program focused on the nature, effects and causes of panic, and the second on useful and non-useful ways of managing panic. Negative selfstatements were discussed as were errors in thinking. Brief techniques on how to overcome these cognitive errors were explained. The program, placed on the web-site of the University of Ballarat whereby participants could access it via a password, was designed to be more engaging and to allow more flexible use than usual texts on panic. It included considerable use of colour, animated illustrations, hyperlinks between sections of the text, and selfassessment quizzes with immediate feedback.

Participants were randomly assigned to either the treatment $(n=11)$ or to self-monitoring only $(n=12)$. At the initial interview participants completed the Body Vigilance Scale, the Anxiety Sensitivity Index and the Self-Efficacy Questionnaire. They were also instructed to record daily panic frequency, anticipatory fear of panic, general anxiety and depression daily for the 3 weeks of the study. Participants in the intervention condition were told that treatment would commence after one week (i.e. beginning of week 2) and the program must be completed within that week. During this second week, they were able to access the Internetbased program at the University of Ballarat or were free to access it from their own terminal source. Participants in the self-monitoring only condition continued to self-monitor in the second week and completed the same questionnaires and record forms as those accessing the computer program.

\section{Treatment of data}

Data on panic frequency, anticipatory fear of panic, average daily anxiety and depression were summed, averaged and collapsed into two separate data points. The first data point corresponded to the average daily ratings in the first week after the initial interview and before the intervention group commenced accessing the program, and the second data point corresponded to the post-treatment phase (i.e., taken during the third week of the study after the program had been completed by the intervention group). Data points for the Body Vigilance Scale, Anxiety Sensitivity Index and Self-Efficacy Questionnaire corresponded to the pre-treatment assessment at the initial interview, and to a post-treatment assessment one week after participants had finished using the program (at the end of week 3). 


\section{Results}

For panic frequency, the condition by time interaction was significant, $F(1,19)=12.63, p<$ .01 , with an effect size of .40 and power of .92 . Within group comparisons found a significant decrease in panic frequency from pre- to post-assessment for the treatment group only, $t(8)=-2.53, p<.05$. For anticipatory fear of panic, the condition by time interaction was significant, $F(1,19)=12.26, p<.01$, with an effect size of .39 and power of .91 . There was a significant pre- to post-assessment decrease for the treatment group only, $t(8)=-3.30$, $p<.05$. For general anxiety, the condition by time interaction was significant, $F(1,19)=$ $8.92, p<.01$, with an effect size of .32 and power of .81 . There was a significant decrease from pre- to post-assessment for the treatment group only, $t(8)=-2.68, p<.05$. For general depression there was a non-significant trend towards a condition by time interaction, $F(1,19)=3.71, p=.07$, with an effect size of .16 and power at .45 .

There was a significant condition effect for self-efficacy, $F(1,19)=13.52, p<.01$ with an effect size of .42 and power of .94 ; a significant time effect, $F(1,19)=9.08, p<.01$ with an effect size of. 50 and power .95 ; and a condition by time interaction, $F(1,19)=6.52$, $p<.05$ with an effect size of .26 and power at .68 . There was a significant pre- to postassessment increase for the treatment group only, $t(8)=-2.92, p<.05$.

There was a significant condition effect for body vigilance, $F(1,19)=4.94, p<.05$ with an effect size of .21 and power of .56; a significant time effect, $F(1,19)=6.61, p<.01$, with an effect size of .42 and power of .86 ; and a condition by time interaction, $F(1,19)=$ $7.91, p<.05$, with an effect size of .29 and power of .76. There was a decrease from preto post-intervention for the treatment group only, $t(8)=4.27, p<.01$. For both groups together, anxiety sensitivity decreased over time, $F(1,19)=7.46, p<.01$, with effect size of .28 and power of .74. No other comparisons were statistically significant.

\section{Discussion}

Results suggested this Internet-based treatment program was an effective intervention for people with panic disorder, at least in the short-term. Specifically, reductions in frequency of panic, anticipatory fear of panic and general anxiety levels were found only for the treatment group. As well, levels of body vigilance decreased and self-efficacy for managing panics increased for the treatment group. However, there were no differential effects of treatment on anxiety sensitivity. Because participants were reassessed only one week after intervention, the results only account for the immediate effects of the intervention and not the possible long-term benefit. Further controlled studies also are required to rule out the possibility that expectancy and demand effects were responsible for the present results.

This study incorporated some therapist-participant involvement in that the senior author needed to show participants how to access and navigate the Internet-based program, and to check that they were accessing it during the active treatment phase. More detailed instructions on the use of the program may have reduced this time but the optimal conditions for delivering these type of interventions require further intervention.

\section{References}

Gould, R. A., \& Clum, G. A. (1995). Self help treatment for panic disorder: A replication and extension. Behavior Therapy, 26, 533-546. 
Hecker, J. E., Losee, M. C., Fritzler, B. K., \& Fink, C. (1996). Self-directed versus therapist directed cognitive behavioral treatment for panic disorder. Journal of Anxiety Disorders, 10, 253 265 .

Newman, M. G., Consoli, A. J., \& Taylor, C. B. (1997). Computers in assessment and cognitive behavior treatment of clinical disorders: Anxiety as a case in point. Behavior Therapy, 28, $211-$ 235 .

Newman, M. G., Kenardy, J., Herman, S., \& Taylor, C. B. (1997). Comparison of palmtopcomputer-assisted brief cognitive-behavioral treatment to cognitive-behavioral treatment in panic disorder. Journal of Consulting and Clinical Psychology, 65, 178-183. 